\title{
Metastatic Malignant Neoplasm in the Gallbladder
}

National Cancer Institute

\section{Source}

National Cancer Institute. Metastatic Malignant Neoplasm in the Gallbladder. NCI

Thesaurus. Code C7482.

A malignant neoplasm that has spread to the gallbladder from another anatomic site.

Representative examples include metastatic melanoma, and metastatic carcinomas

from the breast and the stomach. 